\title{
LALIMENTATION ARTIFICIELLE DE L'ALEVIN DE TRUITE ARC-EN-CIEL . (Salmo irideus Gibb.)
}

par R. LIGNIERES et H. HOESTLANDT

(Laboratoires de Zoologie des Facultés libres des Sciences de Lyon et de Lille)

\section{INTRODUCTION}

Dans un article antérieur de R. Tagand, H. Hoestlandt et L. Eiraud (1947), l'influence de quelques facteurs de croissance a été étudiée chez l'alevin de Truile arc-en-cicl (Salmo irideus GiBu). Cet alevin s'elève assez facilement en laboratoire et peuple la plupart des Salmonicultures framf̧aises ; c'est pourquiri il nous a semblé intéressant de réalisłer de nouvelles recherches au cours des années 1947 et 1948 , en rous limitant au domaine de l'alimentation arlificielle. Les élevages sont réalisés dans des conditions de vie identique, hormis le facteur à éludier; dans ce cas, l'étude comparative des courbes de croissance peut corduire à des conclusions précistes.

Le présent travail comporle d'abord un chapilre décrivant la méthode utilisée, puis trois chapitres sur divers essais det remplacement de lat nourrilure animale par de la nourriture végétale, un chapitre sur l'utilisation du sang, un autre sur le rôle de la levure de bière et de l'huile de foie de morue, et un dernier chapitre sur l'action des vilamines $B_{2}$ et $E$. Un appendice envisage les causes probables des variations de croissance entre les sujets des années 1947 et de 1948. Des conclusions pratiques, applicables en Salmonicullure, terminent cet exposé.

\section{CHAPITRE PREMIER}

\section{ORGANISATION ET CONDUTTE DES EXPERIENCES}

Les expériences se sont échelonnées sur les quatre premiers mois de la vie de l'alevin, période pendant laquelle sa fragilité nécessite des soin attentifs, tant au point de jue de l'hygiène que de l'al'mentation Avant chaque repas, le fond des aquariums est soigneusement dćbarrassé des déjections et des particules alimentaires de la veille ; une fois par semaine, les aquariums sont vidés, brossés énergiquement et passés à l'cau permanganátée à $5 \% / \infty 0$ et les alevins sont soumis au bain formolé a $0,4 \%$ recommandé par Léger (1936). Enfin, une fois par semaine également, du 
fromage blanc remplace la nourriture habituelle. Nous n'avoñs enregiatré au cours des deux années d'expérience que quelques cas d'inflammation du tube digestif (catarrhe intestinal de Léger) dus aux expériences alimentaires et do nombreux cas de mortalite par athrepsie lorsque le succédané utilisé était insuffamment nourrissant ou inassimilable.

Le 7 Février 1947 nous recevons 600 oufs de Salmo irideus, issus d'un même couple de géniteurs et pondus ce jour. Ces œufs noús sont remis par M. Burdin, Inspecteur des Faux et Forêts à Lyon, Directeur de l'Etablisseiment Domanial de Pisciculture de Vizille; nous le remorcions. de son extrême obligeance envers nous.

L'éclosion s'échelonne entre le 16 et le 18 Février. Deux jours aprèy l'éclosion, les alevins sont pesés selon une technique qui sera exposée ultérieurement el nous en registrons comme poids moyen 83 milligrammes par alevin. Le 3 Mars nous pesons à nouvequ un lot de 40 sujets : lo poids moyen est de rog milligrammes.

La vésicule vitelline étant à demi-résorbée, dès le lendémain nous disposons les alevins par lols de 40 dans les aquariums d'expérience.

Chaque aquarium de verre mesurc o m. 30 de longueur, sur $0 \mathrm{~m}$. 25 de largeur ; la hauteur de l'eau est de o m. 18, ce qui équivaut à une contenance de 3,5 litres. Un carton noir est appliqué sur lęs parois latérales pour maintenir les alevins dans une demi-obscurite.

L'eau de la ville de Lyon (eau' du Hhơne, filtrée at legèrement jayellisée) arrive clans chaque aquarium par un robinet qui la laisse s'écouler sous forme d'un mince filet à raison de o, 250 fitre par minute.

Le trop plein est constitué par un tube de verre.central dont lorifice est protégé par une grille très fine.

La température de l eau oscille au début entre 8 et io degrés C. ; elle montera en fin Mai jusqu'à 23 degrés $C$.

Comme l'a préconisé D'Assembounc ( 943 ), nous commençuns à nourrir les alevins dès leur placement en aquariums d'expérience, avant que leur vésicule ne soit complètement résorbée.

Le nourriture est préparée une cois par semaine; elle est mise dans de petits gobelets de celluloïd de couleurs différentes portant le numéno des aquariums auxquels ils correspondent. Pour éviter que les produits ne s'altèrent, ils sont conservés dans un frigidaire à une température de 2 à 3 degrés C. en dessous de zćro. Les vitamines $\mathrm{B}_{2}$ et $\mathrm{E}$, ainsi que l'huile de foie de morue, sont incorporées à la nourriture au moment même des distributions.

Bien que tous les éleveurs recommandent au moins trois rupas journaliers, nous ne nourrissons nos alevins qu'une fois par jour ; notre but n'est pas d'obtenir les sujets les plus gros possible en un minimum de temps, mais de déterminer les valeurs nutritives respectives des produits utilisés. Pour cela, il suffit de comparer les courbes de croissance obtenues en nour- 
rissant les alevins avec un repas unique et abondant. Afin d'être assuré qué tous les sujets ont à leur disposition une ration suffisante, nous étalons journellement sur les grillęs 3 grammes de nourriture dès le début du nourrissage ; cette quantité est progressivement augmentée pour attẹindre ro grammes par jour à la fin de l'expérience.

Cette ration nettement trop forte aurait pu fausser les résultats enregistrés on permettant aux jeunes salmonidés de choisir les constituants des mélanges leur convenant le mieux. Mais la gloutonnerie de ces poissons les a toujours incités à se précipiter sur les grilles de nourrissage dès leur introduction dans l'eau, pour se gaver en quelques minutes de la nourriture qui leur était offerte; de plus, cette dernière était, en général, si homogène, qu'il aurait été difficile à un alevin de n'en ingérer qu'une partie.

Les courbes de croissance des sujets de chaque aquarium sont établies d'après le poids moyen de ceux-ci. Pour réaliser les pesées, nous ulilisons une balance sensible au milligramme. Sur l'un des plateaux nous disposons une boîte de Pétri remplic aux trois-quarts d'eau et munie de son couvercle pour éviter l'évaporation. L'autre plateau porte une tare et nous équilibrons à l'aide de poids marqués. L'ensemble des alevins d'un aquarium est alors introduit dans la boîte de Pétri après que l'épuisette qui les contient a été séchée sur un papier filtre pendant dix secondes. Ncus notons les poids à enlever pour rétablir l'équilibre entre les deux plateaux et nous avons rainsi le poids des alevins introduits, d'où l'on tire facilement le poids moyen d'un sujet.

En 1948, nous reprenons, pour les préciser, certains dẹ nos essais antérieurs tout en continuant à réaliser le programme de nos investigations. Nos expériences débutent les ${ }^{\text {er }}$ et 2 Avril (soił 42 jours plus tard que l'année précédente) selon les mêmes méthodẹs et à l'aide d'un dispositif sensiblement identique, ne comportant que quelques modifications minimes ayant trait à l'agencement des aquariums el au nombre des alevins contenus dans chacun d'eux.

Le 16 Avril, la vésicule vitelline des sujets étant à demi-résorbée, nous les disposons par lot de $3 o$ dans les aquariums après avoir déterminé leur poids moyen qui est de 105 milligrammes et nous commengons à les alimenter.

\section{CHAPITRE II}

\section{LA FARINE DE BLE COMME COMPLEMENT D'UNE ALIMENTATION} A BASE DE PULPE DE RATE.

La rate est utilisée depuis longtemps par les pisciculteurs pour nourrir les jeunes aleving, et si son emploi est universellement répandu, c'est Harce qu'il a été reconnu qu'elle convenait parfaitement aux hesoing des très jeunes salmonidés. Il n'y aurait donc pas lieu de vouloir abandonner 
cet aliment qui a fait ses preuves ; or depuis huit ans on attribue a cet abat une place importante dans la fabrication de charcuteries et de patés réseirvés à l'homme si bien que sa destination s'en est trouvée modifiée. Il a donc fallu que les pisciculteurs s'adressent soit des aliments de remplacement soit à des aliments de complément pour pallier la pénurie ou la mreté de cet organe. Nous nous sommes attachés dans ce chapitre a déterminer dans quelles proportions il était possible de remplacer la pulpe de rate par de la farine de blé. Nous avóns fait cuire cette farine parce qu'après exłamen des fèces d'unè Truitelle de plusieurs mois nourrie avec cette denrée, nous nous étions rendus compte que l'amidon était mieux àssimilé après cuisson.

\section{Histonique.}

Ies auteurs cités au cours de cet historique, ainsi que la plupart de ceux dont les noms sont mentionnés dans les chapitres suivants, n'ont expérimenté que sur des Truites adultes. C'est pourquoi leurs exposés n'ont qu'un intérêt indicatif dans la conduite de nos investignations.

Parmi les travaux traitant de l'alimentation de l'alevin, certains touchent à la substitution d'une partic de la pulpe de rate par d'autres substances, mais aucun n'apporte le résultat d'expériences précises.

Blanchon (igio) rapporte aux expériences de M. Jousset de Bellesme è l'aquarium du Trocadéro l'origine de l'adoption des rates dans les piscicultures et dit qu'après ses expériences comparatives il reconnaît que, en exceptant la nourriture vivante, c'était celle que les alevins acceptaient le plus tacilement lorsqu'ils commençaient à manger.

Cependant dés 1927 Haemper, pour obvier au manque de vitamines de la nourriture succédanée, conseilla d'y ajouter du son.

En 1932, Gaschotr faisant une étude de la nourriture naturelle de la Truite met en évidence sa richesse en vitamines, ainsi que sa grande digestibilité due à l'action des éléments del lest qu'elle renferme.

S'inspirant alors de ces données, de nombreux auteurs, pour favoriser ln digestion de la nourriture artificielle, consetllent d'y ajouter de la sciure de bois dur (hêtre) ou du sable.

\section{Recmenches Personnelles.}

Pour réaliser cette étude quatre aquariums sont utilisés : les alevins du premier servant de témoins sont nourris exclusivement à la pulpe de rate ; ceux des trois autres (II, III, IV) reçoivent une nourriture composée de pulpe de rate et de farine de blé cuite à l'eau (ces éléments étant mélangés dans des proportions différentes comme l'indique le tableau I). De plus, à $\mathbf{k a t i o n}$ quotidienne de chacun de ces trois demiers aquariums, nous ajoutons un pour cent d'huile de foie de morue et deux pour cent de levure de bière. 
Tableau 1 .

\begin{tabular}{|c|c|c|c|c|}
\hline$\frac{1}{a} \cdot$ & Rate & Pate de blé & $\begin{array}{l}\text { Hulle de } \\
\text { foie Morue }\end{array}$ & Levure \\
\hline $\begin{array}{l}\text { Aquarium I (temoin) } \ldots \\
\text { Aquarium II. } \ldots \ldots \ldots \ldots \\
\text { Aquarium III. } \ldots \ldots \ldots \ldots \\
\text { Aquarium IV.. } \ldots \ldots \ldots\end{array}$ & $\begin{array}{r}100 \% \\
72 \% \\
47 \% \\
22 \%\end{array}$ & $\begin{array}{l}25 \% \\
50 \% \\
.75 \%\end{array}$ & $\begin{array}{l}1 \% \\
1 \% \\
1 \%\end{array}$ & $\begin{array}{l}2 \% \\
2 \% \\
2 \%\end{array}$ \\
\hline
\end{tabular}

L'état de santé des alevins nous est donné par le pourcentage des mortalités enregistrées dans chaque aquarium en fin d'expérience.

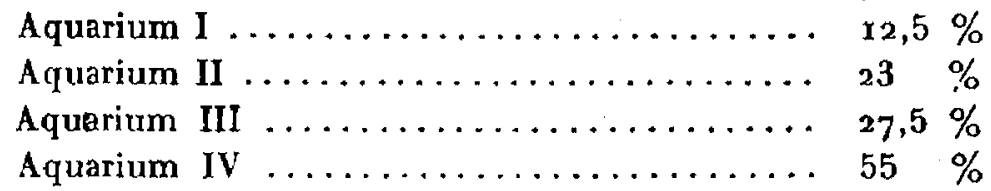

Les résultats des pesées sont notés dans le tableau II ct représentés par le graphique ( $x$ ) qui porte en abcisses les temps à partir de la mise en aquarium et en ordonnées les poids moyens d'un alevin en milligrammes. Pour ne pas surcharger inutilement tableaux et graphiques, les pesés antérieures à la date de la mise en aquarium ne sont pas mentionnées.

Tableau $H$.

\begin{tabular}{|c|c|c|c|c|}
\hline Dates & $\begin{array}{c}\text { Aquarium I } \\
\text { (témoin) }\end{array}$ & Aquarium II & Aquarium III & Aquarium IV \\
\hline 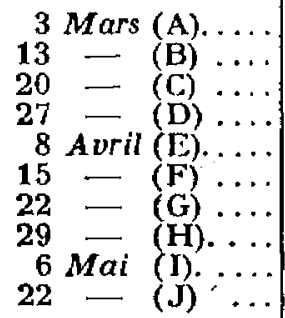 & $\begin{array}{l}109 \\
150 \\
164 \\
158 \\
250 \\
324 \\
405 \\
472 \\
604 \\
862\end{array}$ & $\begin{array}{l}109 \\
154 \\
164 \\
169 \\
257 \\
351 \\
446 \\
554 \\
680 \\
906\end{array}$ & $\begin{array}{l}109 \\
155 \\
159 \\
180 \\
253 \\
329 \\
413 \\
509 \\
575 \\
798\end{array}$ & $\begin{array}{l}109 \\
154 \\
175 \\
187 \\
249 \\
299 \\
\mathbf{3 8 3} \\
\mathbf{4 1 2} \\
\mathbf{4 3 6} \\
\mathbf{4 9 4}\end{array}$ \\
\hline
\end{tabular}

\section{Discusston et interpatetation des pésultats.}

L'observation des courbes de croissance nous apprend que jusqu'au 8 Avril (E), c'est-à-dire 47 jours après leur naissance, les alevins vivant sur leurs réserves vitellines ne nécessitent que très peu de principes nutritifs puisque ce n'est qu'à cette date que les courbes divergent nettement.

A partir de ce moment, nous constatons que lorgqu'un minimum de protides de qualité est assuré, le jeune salmonidé se trouve avantagé par 
une nourriture complète lui apportant protidés, lipides, glucides, vitamines, minéraux el élémenis de lest. En effet, le régime comprenant $72 \%$ de nulpe de rate, $25 \%$ de farine de blé cuite 3 l'eau, $\%$ d'huile da foie de morue et $2 \%$ de levure de bière donne un meilleur résultat que la pulpe de rate pendant la durée des expériences.
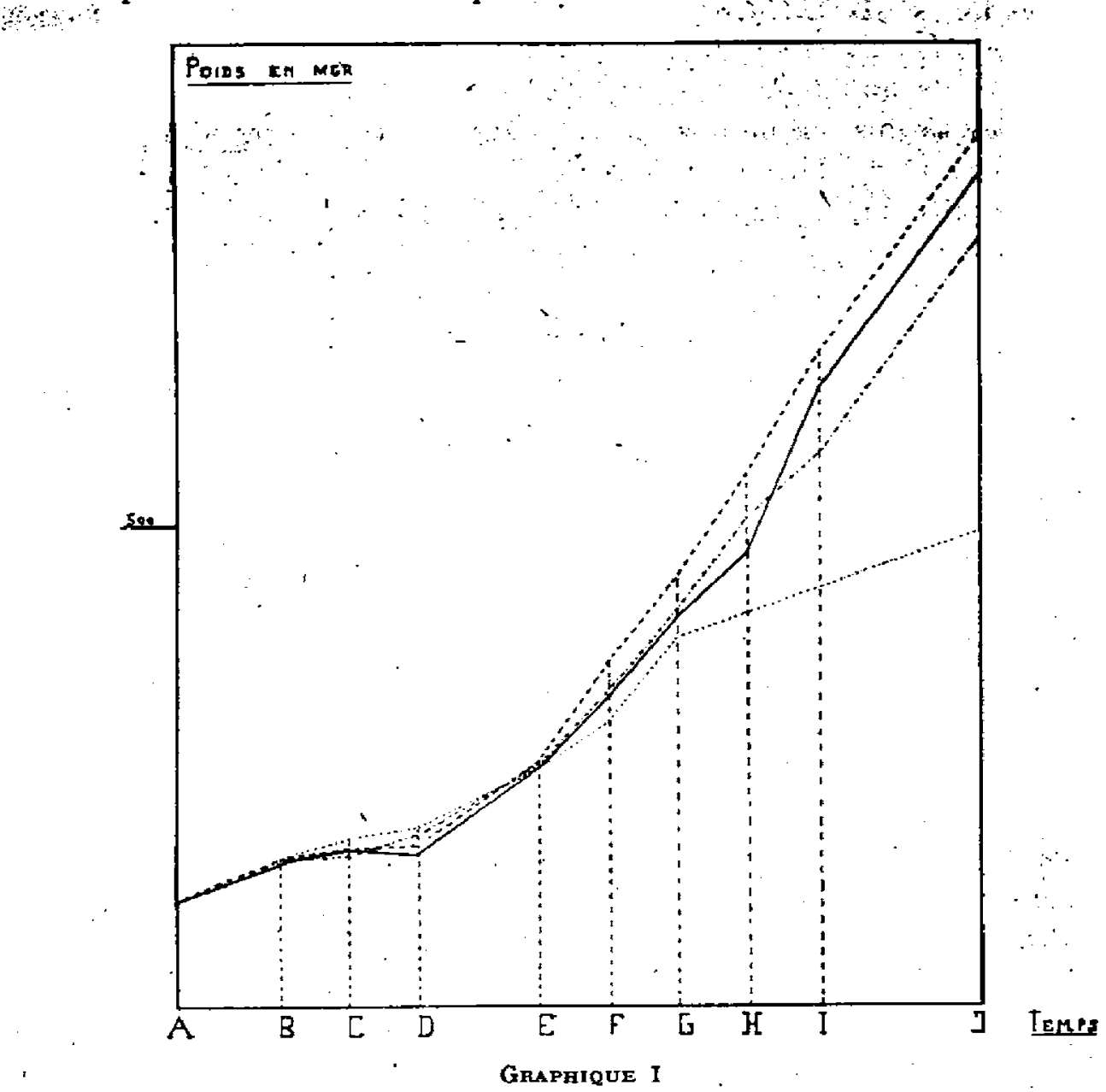

Aquarium I (témoin).

Rate pure.

Rate $72 \%$, Blé $25 \%$, Huile $1 \%$, Levure $2 \%$

Rate $47 \%$, Blé $50 \%$, Huile $1 \%$, Levure $2 \%$.

- - - Aquarium 11.

- . - - . Aquarium III.

Rate $22 \%$, Ble $75 \%$, Huile $1 \%$, Levure $2 \%$.

Le régime comprenant $47 \%$ de pulpe de rato, $50 \%$ de farine de blé cuite à l'eau, r \% d'huile de foie de morua, $2 \%$ de levure de bière, convient mieux que la pulpe de rate pure jusqu'au $7^{\circ}$ jour de la vie de l'alevin.

Enfin le régime constitué par $22 \%$ de pulpe de rate, $75 \%$ de farine de blé cuité à l'eau, I \% d'huile de foie de morue, $2 \%$ de levure de bière est nettemente insuffisant dès le début de la nutrition de I'alevin.

Le pourcentage des mortalités croît de l'aquarium I à l'aquarium IV, et prouve également les effets néfastes d'un régime carencé en protideṣ de qualité, 
 \\ GHAPITRE III \\ ESSAIS DE QUELQUES MÉLANGES ALMIENTAIRES ASSOCIANT DES PRODUITS DIVERS D'ORIGINE ANIMALE ET VEGETALE.}

Stimulés de tout temps par le problème de l'abaissement du prix di revient des alevins, de nombreux auteurs se sont consacrés à la recherch de mélanges alimentaires susceptibles de remplacer les denrées onéreuse: employées jusqu'alors, mais ils ne donnent pas de résultats bien précis Plus près de nous, au cours des périodes de disette que nous venons $d t$ traverser, les pisciculteurs se sont souvent vu contraints d'utiliser des pro duits divers que leur offraicnt les ressources locales pour entretenir leur: alevins, mais beaucoup eurent des déboires ; aussi avons-nous pensé qu’i serait bon d'apporter quelques précisions sur les valeurs alimentaires pra. tiques de certaines denrées courantes, mais peu utilisées d'ordinaire en salmoniculture comme le sont les farines de poisson, de viande, de blé eí les déchets de pâtes alimentaires.

Dans le but d'obtenir une ration d'entretien peu onéreuse, nous avons associé $75 \%$ de produits d'origine végétalc à $22 \%$ de produits d'origine animale, le tout étant complété par l'apport d'huile de foie de morue el de levure de bière.

\section{Historique.}

JuLlerat (192I) dít que les matières animeles séchées réduites en poudre impalpable peuvent être facilement absorbées par les petits alevins, mais il affirme que toutes celles qu'on préconisait et qu'il avaił essa yées donnenf des résultats déplorables, finissant invariablement par détorminer des épidémies qui déciment rapidement les élevages.

Barthélemy el Julin ( 193 r) conseillent aux salmoniculteurs d'ajouter à l'aliment porteur de vitamines le complément d'origine végétale ou le produit commercial qu'ils jugenont convenable ; ensuitei ils devront, par des essais suivis et répétés, rechercher le mélange " au goat " de la Truite ainsi ques le dosage et le mbde de trituration pour qu'il soit pris facilement ct assimilé commodément.

LuUrencin (z934), traitant de l'utilisation des farines de poissons ef salmoniculture, écrit que les avis sont parlagés à leur sujet, certains pisciculteurs en étant très satisfaits à tout point de vue : prix de revient, conservation, pouvoir nutritif, d'autres n'ayant eu que des déboires. Il assure, sans trancher cependant la question, qu'il s'agit là d'un produit d'avenir dont la valeur alimentaire est indiscutable.

HaEmpel et Liepolt ( 1935 ) rapportent une série d'expériences effectuées 
avec trois farines de poisson d'origine différente, mais de même richesse en protides. Ils constatent que la farine I obtenue à partir de certains poissons de mer maigres se révèle excellente alors que la farine II provenan! dautre poissons de mer maigres se montre sans effet appréciable sur la croissance des sujets en expérience. Quant à la farine III tirée de poissons d'eau douce (Pelecus cultratus) dont la teneur en graisse est élevée (r3,02\%) et la minéralisation faible, elle entrấne une forte mortalitś. Ils concluent que la valeur nutritive 'd'un aliment n'est pas suffisamment défini par sa composition chimique et qu'il faut des essais biologiques pour être fixé en toute sécurité.

Ces mêmes auteurs expérimentant avec deux farines de viande, dénommées Carnarina et Karnas, pouvant être qualifiées de bonnes d'après leur analyse chimique, obtiennent un très faible accroissement du poids de leurs sujets et une très forte mortalité. Mais ils constatent une très nêtte amélioration de la croissance dans le cas des farines de poisson par adjonction de farine de riz et, dans le cas des farincs de viande, par padjonction de son de froment ou de proies vivantes. Ils apportent ainsi la démonstration qu'aux farines de poissons et surtout aux farines de viande font défaut certains éléments indispensables à la santé de l'alèvin sans toutefois les préciser.

Barthélemy (1g35) prétend que les déhoires obtenus en utilisant les farines de poisson et de viande ne sont dus qu'à une avitaminose proverant du mode de dessication de ces produits et de la manière de préparcr et de distribuer les patées. Il conseille l'adjonction de son de céréales, de traces d'huile de foie do morue, de déchets d'industrie laitière et il cite Musard qui obtient de bons résultats en fabriquant un pain constitué par un mélange de farines de poissons, de farines de blé ou de seigle ou te riz auquel il ajoute du słng de cheval, de la Ievure ct des déchets de laiteri“. Mais il est regrettable que les proportions respectives de ces produits ne soient pas indiquees.

AnDRÉ (1936) dit que le meilleur moyen de lutler contre l'insuffisance des rates et leur prix élevé est d'utiliser la farine de poissons qui constitue pour les alevins de Truite un aliment très suffisant à condition d'y ajouter de temps à autre un peu de foie et de rate; mais il ne donne aucune précision quant aux résultats obtenus par procédé.

Barthélemy et Musano (1937) eoncluent avec les nombreux expérimentateurs qui les ont précédés que les farines de poissons ne peuvent être employées seules pendant de longues périodes, mais qu'elles peuvent suppléer pour un temps très court au manque de nourriture fratche. Ils souhaitent enfin que des essais portant sur ces farines associées à d'autres denrées alimentaires montrent la possibilité d'obtenir de bons résultats tant au point de vue rendement que prix de revient. 
De nombreux auteurs préconisent aussi une alimentation à base de poissons de mer frais et de rogue. Ce sont là des produits que seuls les pisciculteura à proximité de la mer peuvent utiliser car, pour les autres, leur usage en est rendu trop onéreux par les frais actuels de transport La rogue, par son prix de revient moins élevé, est plus intéressante que le poisson frais. Nous regrettons de n'avoir pu nous en procurer, car il nous semblc que son étude, amorcée, par Cnimiss $(1941)$, a urait gagné à être complétée.

\section{Expéruences personnelles.}

Nous utilisons les aquariums IV, V, VI, VII pour lesquels nous établissons les régimes alimentaires indiqués dans le tableau III :

Tableau III.

\begin{tabular}{|c|c|c|c|c|c|c|c|}
\hline & $\begin{array}{c}\text { Pulpe } \\
\text { de rate }\end{array}$ & $\begin{array}{c}\text { Farine } \\
\text { de ble }\end{array}$ & $\begin{array}{c}\text { Dechets } \\
\text { nouilles }\end{array}$ & $\begin{array}{c}\text { Farine } \\
\text { poisson }\end{array}$ & $\begin{array}{c}\text { Farine } \\
\text { viande }\end{array}$ & $\begin{array}{c}\text { Huile } \\
\text { foie } \\
\text { morue }\end{array}$ & $\begin{array}{c}\text { I.evure } \\
\text { bière }\end{array}$ \\
\hline Aquarium IV .... & $22 \%$ & $75 \%$ & & & & $1 \%$ & $2 \%$ \\
Aquarium V..... & $22 \%$ & & $75 \%$ & & & $1 \%$ & $2 \%$ \\
Aquarium VI.... & & $75 \%$ & & $22 \%$ & & $1 \%$ & $2 \%$ \\
Aquarium VII.... & & $75 \%$ & & & $22 \%$ & $1 \%$ & $2 \%$ \\
\hline
\end{tabular}

Les pâtes ont été fabriquées sans oufs.

La farine de poisson a été préparée a partie de ro kilogrammes de poissons de mer maigres ( $R$ aja) auxquels les arêtes ont été enlevées : il a été obtenu, après une dessiccation de 8 jours à l'étuve à $40^{\circ}, 750$ gnammes. de farine contenant $0,8 \%$ de matières grasses.

La farine de viande est une bonne farine dite fourragère utiliséc pour l'alimentation du bétail.

Du point de vue présentation, ces mélanges alimentaires offrent des aspects très différents et leur tenue dans l'eau varie avec chacun d'eux

Le mélange de pulpe de rate et de farine de blé cuite est très homogène et adhère parfaitement aux grilles verticales utilisées pour le nourrissagé.

Le mélange de pulpe de rate et de déchets de pâtes cuits est beaucoup moins homogène; Tes déchets constitués par des morceaux assez gros, ne peuvent entrer dans les minuscules bouches d'alevins. Aussi à partit, de 29 Avril (H), nous les pilons avant de les faire cuire, le melange obtenu. alors avec de la pulpe de rate adhère parfaitement aux grilles et est facilegi ment ingéré.

Les farines de poissons et de viande ne se mêlant pas intimempat à tîn? farine de blé cuite si bien qu'une fois dans l'eau elles se détachent dêtí 
grilles et tombent immédiatement au fond. Nous avons placé ces farines dans de petites nacelles en grillage fin, ce qui en retarde quelque peu la chute.

Les résultats de nos essais sont notés dans le tablqau IV et représentés par le graphique (II). Nous signalons que la poudre de viande ayant été reçue après le début de nos expériences, nous ne l'utilisons qu'à partir du 5 Avril (F) sur des alevins nourris jusque là à la pulpe de rate.

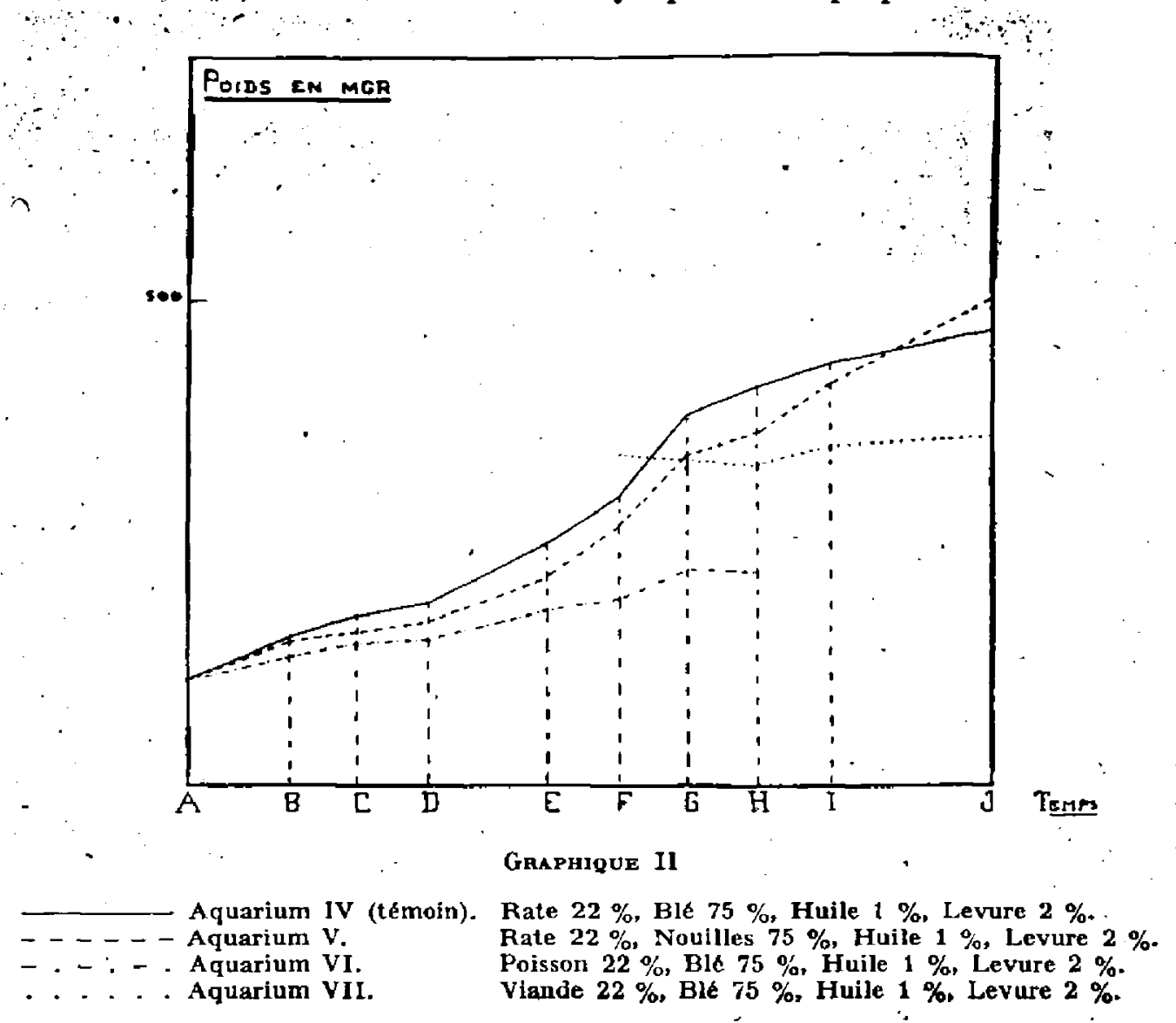

D'autre part, l'expérience réalisée avec la farine de poisson se termine l: 29 Avril (II) : une fuite de l'aquarium (VI) survenue de nuit entraina la mort des alevins par asphyxie.

L'état de santé des jeunes salmonidés au cours de l'expérience nous est donné par le chiffre indiquant le nombre de sujets 'restant dans chaque aquarium à la date de chacune des pesées.

Pour ne pas surcharger le graphique (II) nous ne faisons pas figurer la courbe représentant la croissance des alevins de l'aquarium I nourris a la pulpe de rate pure. D'ailleurs notre but n'est pas de comparer ici la rate pure aux divers mélanges envisagés, młais bien de déterminer la valeur respective des uns par rapport aux autres. 


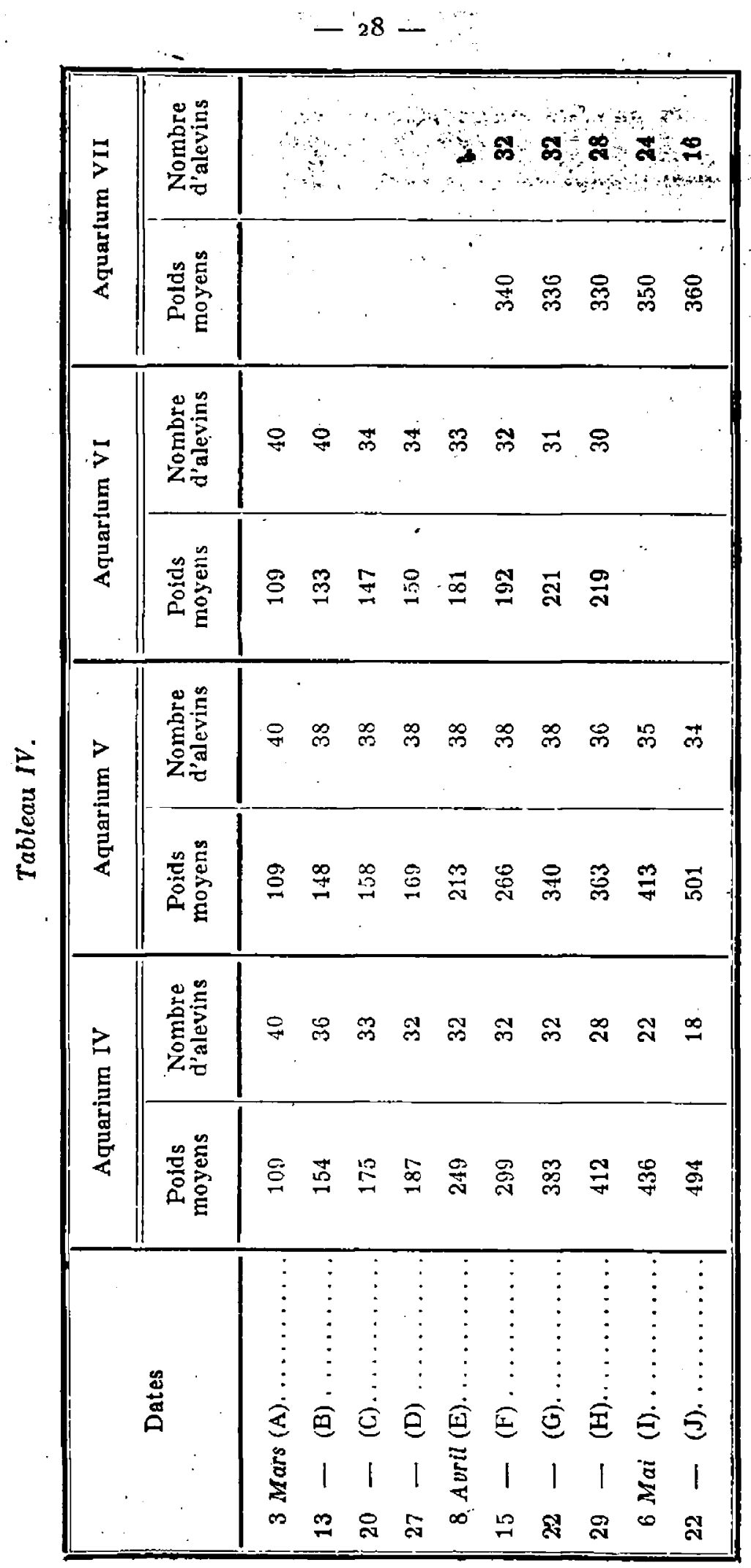




\section{Discugsion et interpé́tation deg Résultats:}

La comparaison entre les résultats obtenus en faisant entrer dans le mélange alimentaire, soit de la farine de blé cuite (IV), soit des déchets de pâtes pilés et cuits (V), nous montre que le premier del ces' oroduits semble conférer une croissanse plus rapide à l'alevin, mais nous devons rappeller ici que jusqu'au 29 avril (H) les déchets de nóvilles non pilés ne sont guère ingérés par les sujets qui doivent se contenter pratiquemènt des $25 \%$ de pulpe de rate que comporte lefur ration. Or, dès cette date, la pulvérisation préalable des débris les faisant réellement incorposer a l'alimen-: tation des jeunes salmonidés, nous constatons un très net redressement de leur courbe de croissance qui ne tarde pas à dépasser celle des aleving recevant la nourriture à base de farine de blé.

De plus il est à remarquer que les sujets de l'aquarium $\mathrm{V}$ ont une meilleure santé puisque le pourcentagre des mortalités n'est que dé i5 \% au lieu de $55 \%$ quand la nourriture renferme de la farinc de blé.

Comme les pâtes alimentaires ne sont en somme qu'une pâte de farine de blé, passée au four après un certain temps de fermentation, il est fort probable que l'influence favorable enregistrée sur la croissance des alevins n'est dụe qu'aux transformations de la pâte de farine de blé provoquées par la fermentation.

En ce qui concerne l'utilisation des farines de poissons.(VI) el de viande (VII). nous enrcgistrons les mèmes résultats négatifs que la majorité des auteurs qui les ont expérimentees ałant nous ; pourtant nous avions eu soin de nous adresser à une farine de poissons de mer maigres dont la température de dessiccation n'avait pas dépassé $110^{\circ}$ et à une farine de viande de bonne qualité. De plus, dans les deux cas, nous avions introduit une importante quantité d'oligo-éléments indispensables, par l'apport d'huile de foie de morue et de levure de bière.

Faut-il conclure que ces produits ne peuvent être utilisés pour nourrir de très jeunes salmonidés; nous ne croyons pas, car il semble que si les alevins n'en ressentent pas les effets c'est parce qu'ils répugnent à aller manger sur le fond ces particules désagrégées. Il suffirait donc de trouver un mélange susceptible de conserver son homogénéité un certain temps dans l'eau pour avoir l'espoir d'obtenir les mêmes résultats favorables que ceux qui ont été en registrées dans l'élevage de la plupart des espèces animales favec des produits similaires.

En définitive, il ressort de cette expérimentation que lẹs déchets de pâtes alimentaires, remplacent avantageusement la farine de blé dans l'àlimentation de l'alevin pourvu qu'on prenne la précaution de les pulvériser finement.

$\mathrm{D}^{\prime}$ autre part, les farines de poissons el de viandes donneraient probablement de bons résultats si l'on parvenait à les incorporer intimement à une nourriture vitaminée au moyen de liants appropriés.

(A sufve.) : 